\title{
Novel design features of the Baby MIND detector for T59-WAGASCI experiment
}

\section{Saba Parsa*}

University of Geneva

E-mail: saba.parsa@unige.ch

\section{On behalf of the Baby MIND Collaboration}

\begin{abstract}
Baby MIND is a magnetized iron neutrino detector, planned to serve as a downstream magnetized muon spectrometer for the WAGASCI experiment [1], on the T2K neutrino beam line in Japan. Access restrictions in its intended location in Japan drove a completely new design of the magnetic modules, a departure from the traditional toroidal magnetized iron detectors such as CDHS and MINOS [2]. The iron plates are magnetized individually by aluminum windings, resulting in a highly optimised magnetic field map. This ensures low power consumption while reaching a uniform magnetic field of $1.5 \mathrm{~T}$. The particle tracking is made by staggered scintillator bars, read out through wavelength-shifting fibers and MPPCs (Multi-Pixel Photon Counters), providing an efficient and compact design. The design allows considerable modularity and is a key to the improvement of charge identification for momenta below $1 \mathrm{GeV} / \mathrm{c}$, not usually addressed by previous detectors of this type. The design of the detector is described and preliminary results of its performance on the beam tests are presented. Baby MIND will provide measurements of charge and momentum for muons from neutrino and anti-neutrino interactions in WAGASCI's water and scintillator cells, at $1.5^{\circ}$ off axis angle to the $\mathrm{T} 2 \mathrm{~K}$ neutrino beam, where the energy of the neutrinos is $\sim 200 \mathrm{MeV} / \mathrm{c}$ higher than that of $\mathrm{T} 2 \mathrm{~K}$ (with $2.5^{\circ}$ off axis angle). By comparing the (anti)neutrino cross-sections on Water with respect to scintillator, and the (anti)neutrino energy reconstruction, these measurements will reduce uncertainties relevant to (anti)neutrino oscillations, especially for CP violation.
\end{abstract}

The 19th International Workshop on Neutrinos from Accelerators-NUFACT2017

25-30 September, 2017

Uppsala University, Uppsala, Sweden

\footnotetext{
*This work is supported by H2020 project AIDA-2020, GA no.654168.
} 


\section{Introduction}

The Baby MIND project was approved by the CERN Research Board in December 2015 [3], the construction phase was completed in June 2017 after 18 intense months, followed by beam tests in June-July 2017 at T9 beamline at CERN. The detector left CERN for shipment to Japan in October 2017, and is planned to be installed at the B2 floor of ND280 complex at J-PARC (Fig1) in January- February 2018 as a muon spectrometer facility for primarily the WAGASCI experiment and in the future for other possible targets that could replace WAGASCI modules. After installation in J-PARC, Baby MIND will enter the commissioning phase with T2K anti neutrino beam until the end of May 2018. During this period, stable data taking with WAGASCI will be practiced. Physics data taking will resume in January 2019, after T2K shotdown for the Super-K gadolinium upgrades.

The physics assignment of WAGASCI and Baby MIND experiment (T59) is to study NeutrinoNucleus interactions at a different off-axis angle than that of the ND280 detector and therefore a slightly different beam energy spectrum. Comparison of the two measurements can help understanding contributions from different neutrino energies, which continues to be one of the difficulties of accelerator based neutrino oscillation experiments as the neutrino beam is non-monochromatic. The target for neutrino interactions is a 3D grid-structure plastic scintillator detector filled with water. The muons from the charged current interactions can be detected by the side MRDs or Baby MIND for momentum determination by range and, in the case of Baby MIND, charge identification and magnetic momentum determination. The charge identification is important to identify the wrong sign backgrounds specially in the antineutrino beam mode since the neutrino contamination is as high as $30 \%$. These measurements would greatly improve the understanding of neutrino interaction at around $1 \mathrm{GeV} / \mathrm{c}$ and contribute to reduce one of the most significant uncertainties of the T2K experiment.
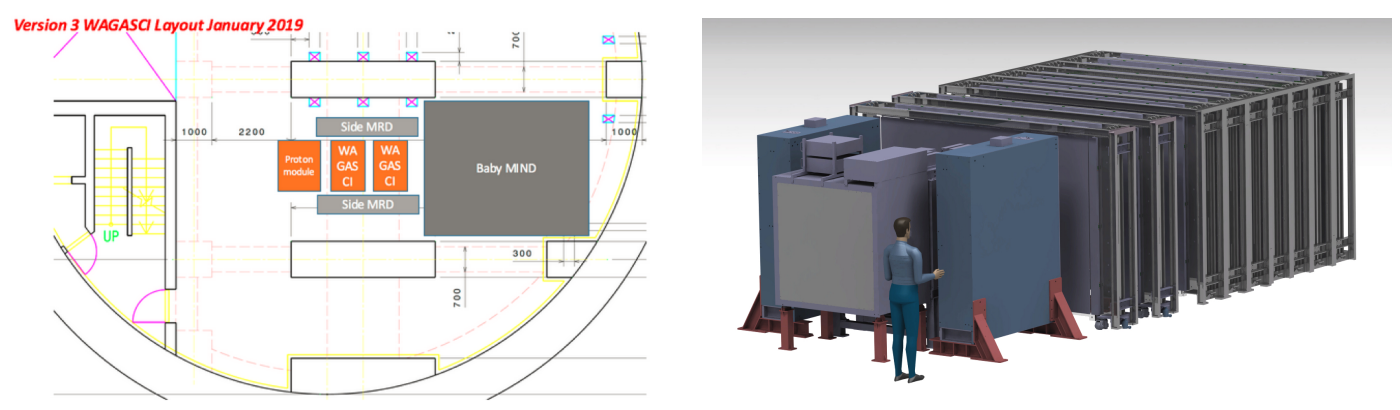

Figure 1: The WAGASCI and Baby MIND detector layout for January 2019.

\section{Detector Hardware}

The design and construction of the Baby MIND detector was very much constrained by the need to operate the detector both at CERN and in Japan on a relatively short timescale, and also the installation limitations in Japan via a narrow shaft, which in particular has driven the new magnetization scheme of the detector. 

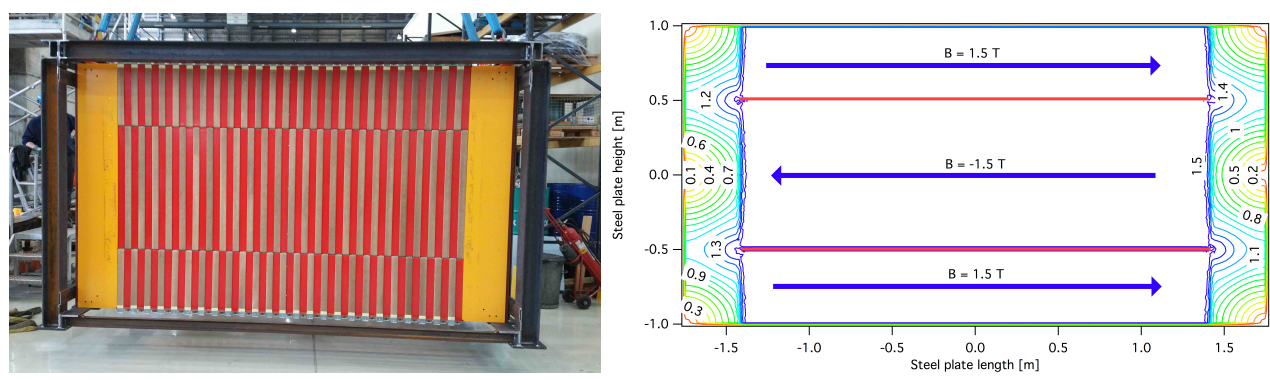

Figure 2: Magnet module (left), Magnetic field map(right).

\subsection{Magnet Modules}

The magnet modules weighing each nearly 2 tons, are made of ARMCO steel with two slits in the middle, and are individually magnetized by Aluminum windings assembled around them in a sewing pattern [4]. This simple and elegant design is a departure from traditional layouts for magnetized iron neutrino detectors (e.g. CDHS and MINOS) which tend to be monolithic blocks with a unique pitch between consecutive steel segments and large conductor coils threaded around the whole magnetic volume. The magnetic field contours for the magnet modules are shown in Fig.2. Homogeneous 1.5 T magnetic field has been measured for all 33 magnet modules. The total power consumption of the magnet system is $11.5 \mathrm{~kW}$.

\subsection{Scintillating Modules}

The scintillator modules with dimensions of $2 \times 3 \mathrm{~m}^{2}$ contain 95 horizontal bars and 16 vertical bars each, with an overlapping pattern to provide the full coverage of the surface of interest and better position resolution. The bars are extruded scintillating plastic with a wavelength shifting fiber, manufactured under the responsibility of INR [5][6]. The light emitted by a particle passage is collected by the fiber and is read by two photosensors (Hamamatsu MPPC) placed at the ends of a wavelength shifting fiber. The horizontal bars are $2880 \times 31 \times 7.5 \mathrm{~mm}^{3}$, with a wavelength shifting fiber along the length of the bar. The vertical bars are $1950 \times 210 \times 7.5 \mathrm{~mm}^{3}$, with a Ushaped wavelength shifting fiber and two MPPCs both reading the signals from the top of the bar (Fig.3). Each module weighs about $400 \mathrm{~kg}$.
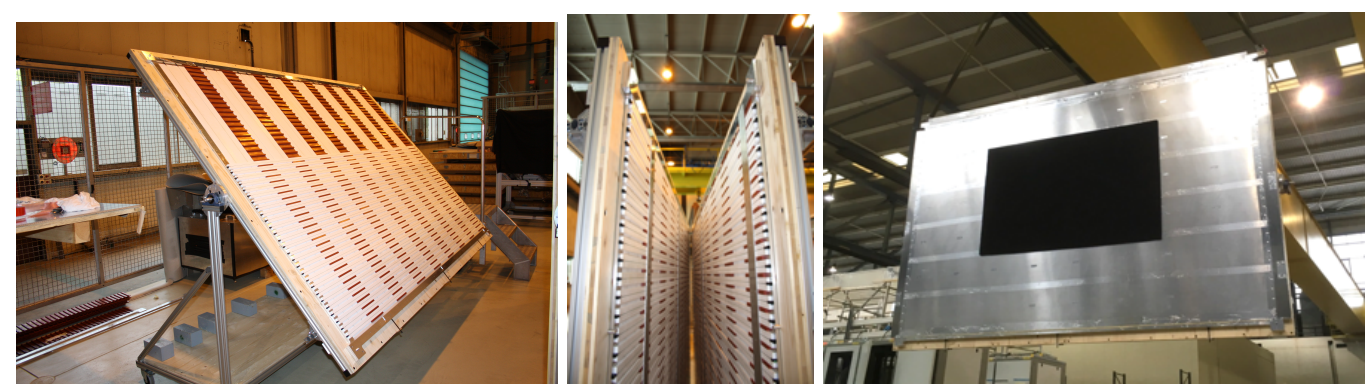

Figure 3: One half of a scintillating module being assembled (left), Two half modules are being brought together (middle), Finished Scintillating module covered by Aluminum cover (right). 

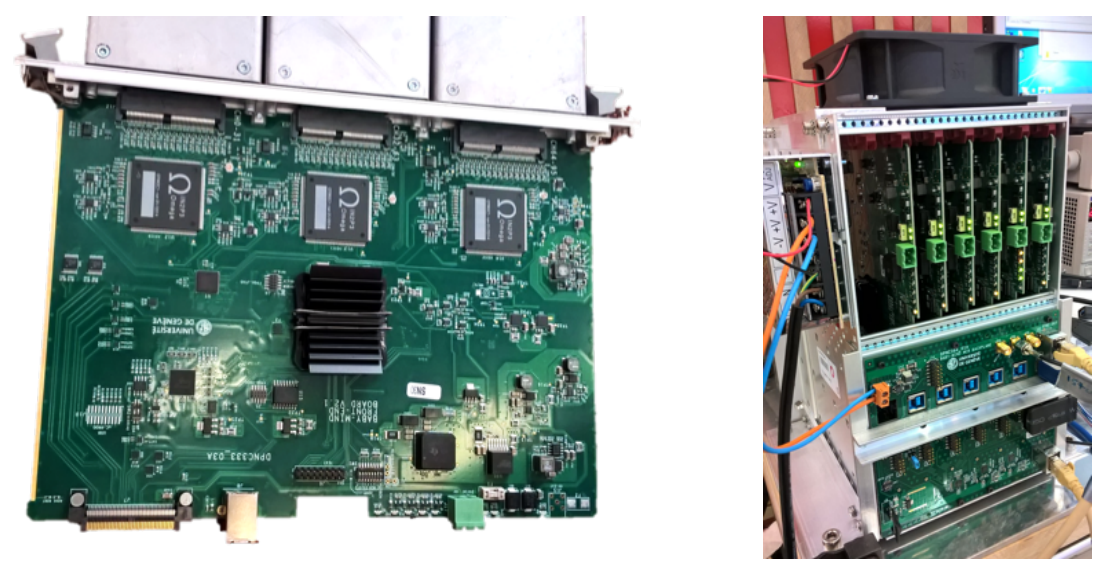

Figure 4: The Front-End Board (left), Mini crate hosting 6 chained FEBs via the backplane (right).

\subsection{Electronic Readout}

The Electronic Front-End boards (FEB), shown in Fig.4, are the main units of the readout system, and are developed by the University of Geneva [7]. Each FEB can process the signals of up to 96 channels through 3 CITIROC chips and an Altera ARIA5 FPGA and push out the data via USB-3 links. The signals from the MPPCs will be combined into bunches of up to 32 channels and will be transferred to the FEBs via custom made cable bundles. Special Mini crates (MCR) were designed to support the FEBs mechanically and to chain up to 6 FEBs via the Backplane board (Fig.4). The final readout architecture also includes also the Master Clock Board (MCB) which is under development by INRNE (Bulgarian Academy of Sciences) collaborators, and will enable synchronization with the WAGASCI experiment.

\section{Beam Tests June-July 2017 at CERN}

Baby MIND beam tests took place at the T9 beam line at the PS experimental hall (East Area),

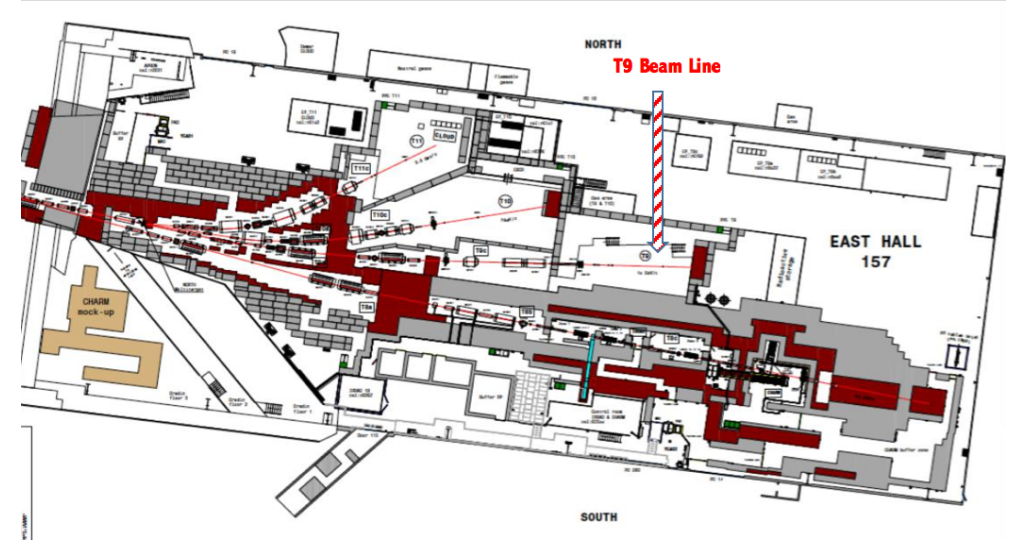

Figure 5: East area and $\mathrm{T} 9$ beam line. 
during June - July 2017. The East Area contains four beam lines: T8, T9, T10 and T11(Fig.5). The beam lines are derived from the $24 \mathrm{GeV} / \mathrm{c}$ primary proton beam from the PS, which provides $2.4 \mathrm{~s}$ cycles of about $400 \mathrm{~ms}$ spill duration. The T9 beam is a secondary beam from the collision of the proton beam with a $200 \mathrm{~mm}$ think Aluminum target, that delivers secondary particles up to $15 \mathrm{GeV} / \mathrm{c}$ at a production angle of 0 degrees. The line is designed to provide the users with non-separated secondary particles, with positive or negative polarity. Users can adjust the beam momentum by setting the currents for the optical magnets of the beam line.

\subsection{Detector layout in the beam test}

In order to support the magnets and scintillating modules mechanicaly, four support frames have been designed and constructed, specially to meet the transport requirements within CERN and while shipping to Japan. Baby Mind modules were installed in the four frames with the layout that is shown in Fig.6. Each block weighs about 20 tons which is within the operational range of the cranes at CERN and within limits for the transport containers to Japan.

A few meters upstream of the Baby MIND modules, AIDA modules were installed. These modules belonging to the project Totally Actice Scintillator Detector (TASD)[9], are built from the same type of scintillating bars with $1 \mathrm{~cm}$ width, and same photosensors as Baby MIND. The particle signals in AIDA modules provide trigger signals for the arrival of beam particles, and also provide information about the incoming angle of the particles. A total of 44 FEBs mounted on 8 mini crates instrumented the 3996 channels of Baby MIND and 192 channels of AIDA.

All 44 FEBs were synchronized using the spill signal from T9 beam line which arrives one second before the arrival of the spill of particles. This signal was fed to a dedicated Master FEB on a separate MCR. The Master FEB would generate a reference clock signal for all the other FEBs acquiring data. The clock signal was distributed to 8 MCRs via three Fanout boards [8].

\subsection{Preliminary results of the beam test}

During the beam tests, many data sets with different beam settings, including positive and negative beam polarity with momenta between 0.5 to $5 \mathrm{GeV} / \mathrm{c}$ were acquired. The detector performance as well as the $\mathrm{T} 9$ beam line properties can be studied by analysing the beam test data. In the results shown in this section, particle hits are constructed by requiring coincidences between

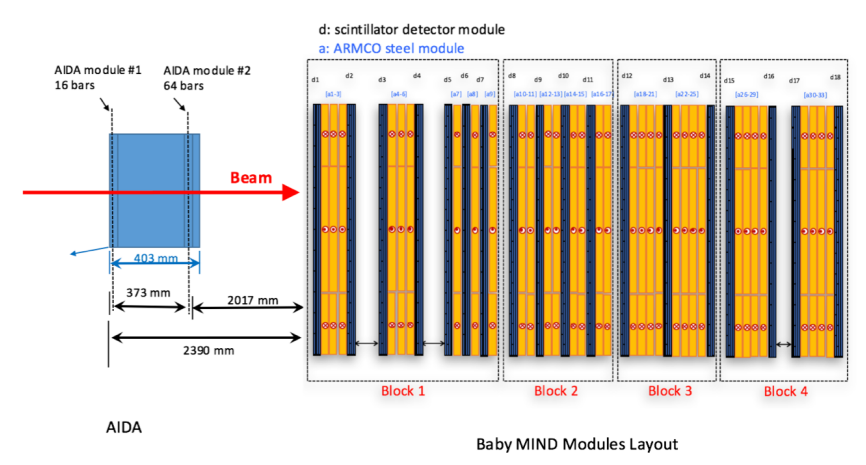

Figure 6: Baby Mind set up at T9 beam line side view. 

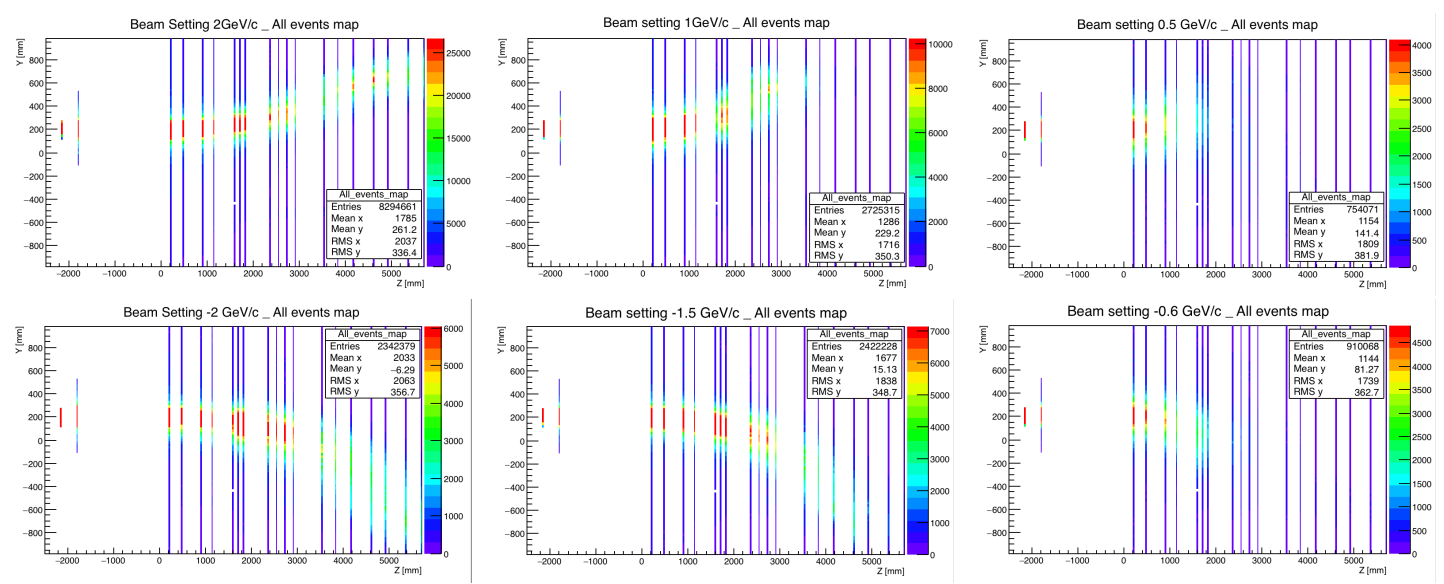

Figure 7: Y-Z profile of hits in detector modules for positive muon beams of $2 \mathrm{GeV} / \mathrm{c}$ (top left), $1 \mathrm{GeV} / \mathrm{c}$ (top middle) and $0.5 \mathrm{GeV} / \mathrm{c}$ (top right) where the particles are bent upwards and negative muon beams of $-2 \mathrm{GeV} / \mathrm{c}$ (bottom left), $-1.5 \mathrm{GeV} / \mathrm{c}$ (bottom middle) and $-0.6 \mathrm{GeV} / \mathrm{c}$ (bottom right) where the particles are bent downwards.

signals of the channels reading the ends of the same horizontal bar within a narrow time window of $12.5 \mathrm{~ns}$. No additional selection criteria other than AIDA trigger signal has been applied. The plots in Fig.7, displaying the side view of the hits in the detector modules, demonstrate the concentration of hits along the particles path as the tracks are bent by the magnetic field. The upper row shows positive beams of $2 \mathrm{GeV} / \mathrm{c}, 1 \mathrm{GeV} / \mathrm{c}$ and $0.5 \mathrm{GeV} / \mathrm{c}$ and the lower row demonstrate negative beams of $-2 \mathrm{GeV} / \mathrm{c},-1.5 \mathrm{GeV} / \mathrm{c}$ and $-0.6 \mathrm{GeV} / \mathrm{c}$.

\subsubsection{Momentum reconstruction from range}

As seen in the plots of Fig. 7, the particles with low momenta are stopped in the middle of the detector modules. We have reconstructed the momentum by range, considering the depth of
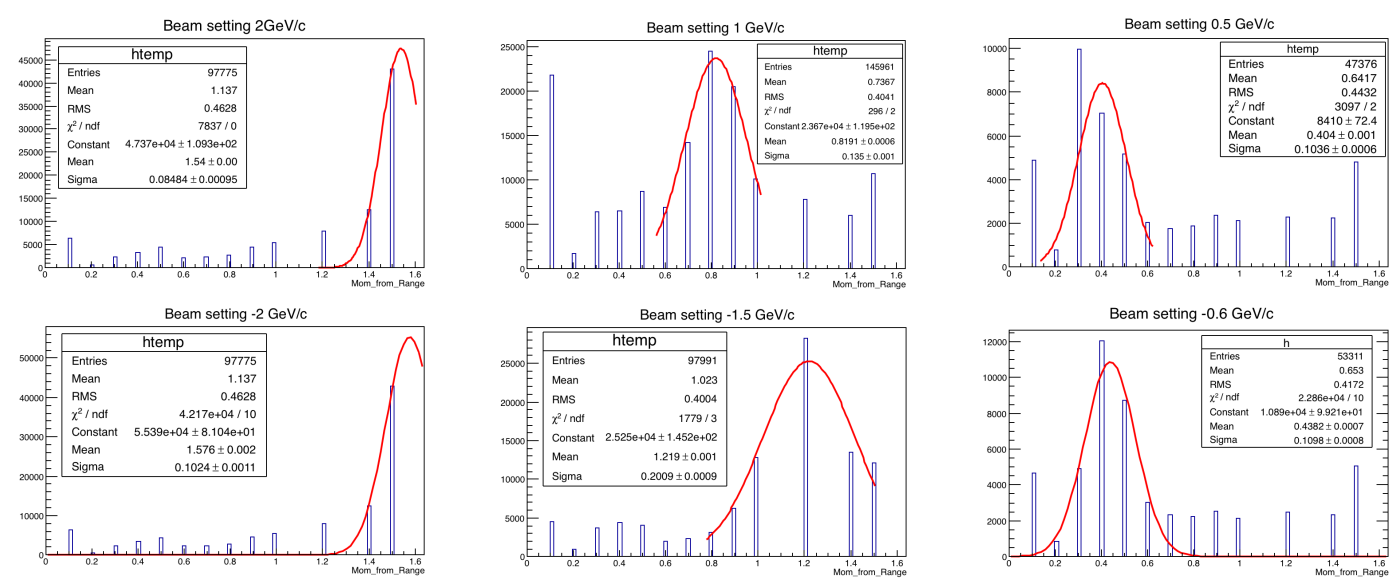

Figure 8: Preliminary mometum reconstruction from range for positive beams (upper row) and negative beams (lower row). The beam momentum decreses from the left plots to the right plots. 
steel and plastic before the last hit of the track, while neglecting the curvature due to the magnetic field. The plots in Fig. 8 show the results of this study. A gaussian fit has been applied to the most relevant part of the data sets, expected to be mostly populated by muons. For the cases of \pm 2 $\mathrm{GeV} / \mathrm{c}$ beams, the fits were forced as the particles escape the detector and only half of the gaussian distribution is available. This method clearly underestimats the actual momentum of the particle tracks. For the case of $1 \mathrm{GeV} / \mathrm{c}$ beam settings, the mean value of the fitted gaussian distribution is $0.82 \mathrm{GeV} / \mathrm{c}$. The reason is that the effective length of a bent track is larger than the thickness of layers, and also because after the last scintillating module the track might still penetrate into a few more magnet modules which we are blind to. A more precise momentum reconstruction algorithm based on curvature and irregularities of distances between consecutive magnetized steel segments is to be developed.

\section{Summary}

The Baby MIND detector has been successfully constructed and tested at CERN and has been transported to Japan. The installation and commissioning of the detector is expected to take place in the first and second quarter of 2018 and physics data taking with the WAGASCI detector will start in January 2019. The novel features of the design and a short description of the main detector components has been presented. The magnet performance during the beam tests at CERN, has been demontrated and preliminary results of the momentum reconstruction from range have been shown. A more detailed analysis of the beam test data is ongoing and will be reported in dedicated publications.

\section{References}

[1] E. Noah et al., "The WAGASCI experiment at J-PARC to measure neutrino cross-sections on water", PoS EPS-HEP2015 (2015) 292.

[2] I. Ambats et al. (MINOS Collaboration) "MINOS Technical Design Report", NUMI-L- 337, FERMILAB-DESIGN, 1998

[3] M. Antonova et al., "Status report for the Neutrino Platform NP05 - Baby MIND - experiment", CERN-SPSC-2017-015, SPSC-SR-210, (2017).

[4] G. Rolando et al. , "New and Optimized Magnetization Scheme for the Baby Magnetized Iron Neutrino Detector at J-PARC", IEEE Transactions on Magnetics, May 2017, Volume 53, Issue 5.

[5] M. Antonova et al. , "Baby MIND: A Magnetized Segmented Neutrino Detector for the WAGASCI Experiment", JINST 12 (2017) no.07, C07028, e-Print: arXiv:1705.10406

[6] W. Baldini et al. , "Measurement of parameters of scintillating bars with wavelength-shifting fibres and silicon photomultiplier readout for the SHiP Muon Detector", JINST 12 (2017) no.03, P03005, e-Print: arXiv: 1612.01125

[7] E. Noah et al. , "Readout scheme for the Baby MIND detector", PoS PhotoDet2015 (2016) 031.

[8] M. Antonova et al. , "Synchronization of the distributed readout frontend electronics of the Baby MIND detector", ISBN: 978-1-5386-1753-3, IEEE Xplore, 10.1109/ET.2017.8124369 (2017)

[9] R. Asfandiyarov et al. , "Proposal for SPS beam time for the baby MIND and TASD neutrino detector prototypes", (2014), e-Print: arXiv:1405.6089 